CYBERNETICS AND INFORMATION TECHNOLOGIES • Volume 15, No 6 Special Issue on Logistics, Informatics and Service Science

\title{
Structural Modelling and Deceleration Algorithm for a Follow Aircraft on Performance-Based Navigation Airway Based on Multi-Agent Technique
}

\author{
Ming Zhaohui ${ }^{1,2}$, Zhang Ming ${ }^{1,3}$, Tang Xinmin ${ }^{1,3}$, Han Song-Chen ${ }^{4}$ \\ ${ }^{1}$ College of Civil Aviation, Nanjing University of Aeronautics \& Astronautics, Nanjing, 210016 China \\ ${ }^{2}$ Mathematics \& Information Technology College, Jiangsu Second Normal University, Nanjing, \\ 210013 China \\ ${ }^{3}$ Aeronautical Laboratory, University of South Florida, Tampa, FL 33620, USA \\ ${ }^{4}$ School of Aeronautics \& Astronautics, Sichuan University, Chengdu, 610065 China \\ Email: hansongchen@nuaa.edu.cn
}

\begin{abstract}
Considering the operation characteristics of the high-speed PBN airway, we propose an aircraft agent structural model and a basic algorithm flow of decision-making by Multi-agent technique. According to the constraint of safe separation and speed with the aircrafts, making use of the car-following theory, the deceleration constraint model is built for a flight management system. When the front aircraft decelerates, the model can offer a speed adjustment proposal for the following aircraft. The model is built based on the minimal safe separation and speed interval constraint; each variable, influencing the deceleration of the following aircraft can be analyzed. Simulation analysis is carried out for different combinations of aircraft types, initial speed, safe separation and deceleration. The follow aircraft deceleration was calculated under different conditions and the results coincided with reality. It is proved that the model can provide safe separation between the two aircrafts.
\end{abstract}

Keywords: Performance-based navigation airway, aircraft agent, structural modeling, follow aircraft, deceleration constraint algorithm.

\section{Introduction}

Performance-Based Navigation (PBN) technique [1] is the latest flight technology developed by the International Civil Aviation Organization (ICAO). It combines satellite navigation with airborne equipment to provide an accurate and safe flight method and an efficient mode of the air traffic management for an aircraft during the whole journey. PBN airway can enlarge the airspace capacity to ensure the flight flow as larger as possible [1, 2]. A larger flight flow means limited 
independence of a single aircraft; a phenomenon similar to the ground traffic flow will occur.

Consequently, the scholars began to use the traffic flow theory for reference in the study of the flight characteristics and capacity of the aircraft. Tandale, Hansen and others (see [3-6]) have established a queue model to describe the traffic flow behaviour at limiting capacity in order to analyze and forecast the delay features of the airspace system and measure the performance of the air traffic management. Bayen, Raffard and Tomlin [7], Robelin et al. [8] have set up a fluid mechanics model to find out the spread law of the traffic flow between sections and to optimize the control measures by using the traffic LWR (Lighthill-WhithamRichards) equation [9, 10]. Men on, Sweriduk and Bil imoria [11], and $\mathrm{Z}$ han g et al. [12] have constructed an evolution/transmission rule of the cellular state to deduce the state transition process of the traffic flow through characterizing the interactions of cells. Z h ang [13] proposed a collaborative control architecture of the traffic flow management for an airport terminal area. Agent technology can also be used in the research of automated control of an aircraft. In [14] the authors constructed a framework for an Air Traffic Flow Management (ATFM) system based on agent technology, illuminated the operational mode of the system and the structure of every agent subsystem. References $[15,16]$ studied the Multi-Agent technology in flight conflicts, through airspace resource modelling - a conflict resolution algorithm is proposed to explore the distribution of the airway resources. $\mathrm{W}$ ang and Xu [17], Wang, Xu and Zhang [18] and Zhang and $\mathrm{Hu}$ [19] proposed tertiary structure of ATFM system based on multi-Agent, designed the internal structure of the system. Hex m o or and Hen g [20] designed a decision support agent system of the tower controller to assist the flight landing and conflict avoidance, the controller agent being responsible for recommended measures and issued relevant instructions. Nits ch ke [21] defined a route agent, an approach agent and a tower controller agent collaborate work, implementing conflict detection and resolution. C a ll antine [22] established a controller agent system based on the behaviour of the aircraft pilot, in order to study how to implement the automated control of a complex air traffic control (ATC) system, and assess the operation of the new air traffic management system. Nguyen, Briot and Drogoul [23] proposed the application of the controller and aircraft agent coordination techniques in the air traffic control, established the framework to reassign the responsibilities and workload of the controller. Hill et al. [24] studied the distributed ATC system of "free flight", the aircraft agents resolved the conflict detection and resolution itself through satisfaction decision theory. A g o g in o and Tumer [25] proposed an operational feedback system to improve the operating efficiency of the overall air traffic system, combining a fixed positioning agent and controllers' agent by decision making mechanisms.

We introduced fly-following characteristics of the aircraft on PBN airway and analyzed flying targets of the aircraft agent. We proposed architecture of the aircraft agent model. The front and following aircrafts on PBN airway are considered. The coordination reasoning rules of the aircraft agent on a PBN airway are defined, the simulation algorithm flow and the constraint conversion equation of the 
deceleration state are established. The deceleration constraint model to be used in an automatic flight management system in the aircrafts is built based on safe separation constraints and a speed constraint. This model can calculate the deceleration based on the minimal safe separation and the speed constraint for the following aircraft, when the front aircraft is decelerated. The model is then subjected to simulation analysis under different combinations of the aircraft types, initial speed, safe separation and deceleration. The results show that the model can ensure safe separation between the two aircrafts.

\section{Flight characteristics of the following aircraft on PBN airway}

PBN airway has the following features [1, 2]: the aircraft is equipped with such precision-positioning equipment as satellite-based navigation and the performance tends to homogenization and can be put under exact control on a $4 \mathrm{D}$ track; the airways are categorized: aircrafts of poor performance are not allowed to fly in advanced airways and the space between the aircrafts has been narrowed; the aircraft flow is of high density, so that the aircrafts are forbidden to go up or down freely. PBN airway is a one-way and single-layer route without crosses where limitations of the performance and high density flight are required. Its control rules include: the controllers to have a uniform command and coordination, while the pilots control the aircrafts; follow aircraft is not allowed to exceed the front aircraft unless there are any special reasons, nor any aircraft in the queue is allowed to lag behind; acceleration and deceleration can be known by the follow aircraft.

The aircraft density is usually high on the PBN airway and the aircrafts are not allowed to descend or ascend freely. Thus, the aircrafts are in a car-following situation. However, when one aircraft on the PBN airway is decelerated suddenly and reports this situation to the air traffic controllers, the following aircraft will undergo an emergency deceleration to ensure safe separation. This change of movement of the front aircraft is considered a stimulus to the following aircraft, which, upon receiving this stimulus, will make a relevant response. This "stimulusresponse" relation is another typical feature of car-following model, which can be used to derive the flight status of the following aircraft from the separation distance between the two aircrafts and the speeds of the two.

In this flight pattern, the air traffic controllers command and coordinate the air traffic, and the pilots are responsible for flying the aircrafts. The pilots will not directly decelerate unless operating under visual flight rules or in some special situations. Hence, the flight following mainly involves the participation of the air traffic controllers, and the aircraft decelerates to a speed above the stalling speed.

\section{An aircraft agent model and a basic algorithm flow of decision making}

The agent receiving broadcast information of the adjacent aircraft is to achieve the situational awareness of the surrounding airspace traffic through Automatic Dependent Surveillance Broadcast (ADS-B) technology. The front aircraft as the 
main object of interest, automatically adjusts the spacing with the help of the received state parameters to ensure the flight safety. At the same time, as soon as possible it must fly by the relevant PBN airway. There must be established any mechanism to achieve safe separation of the aircraft and management of PBN airway by intelligent agent technology.

\subsection{Aircraft agent architecture module}

The main module of the aircraft agent includes: a flight planning module, a position detection and processing module, a conflict detection module, an inference decision-making module, a flight status module and an information interaction module. The surrounding traffic situation shown by the aircraft situation display in real time, provides situational awareness for the pilots. Providing hardware support for the aircraft operation on a PBN airway; the architecture is shown in Fig. 1. The basic module consists of the six modules.

- A flight planning module: includes aircraft, traffic flow and PBN Airway structural features database and flight behavior planner. Aircraft feature database stores the operating characteristic feature information. The traffic flow feature database stores the traffic flow characteristic information. PBN airway feature database stores the spatial structure of the environmental information. The flight behavior planner establishes an aircraft operational control model according to the aircraft characteristics, provides data for the inference decision making module.

The module collects the aircraft and traffic flow characteristic data, extracts information from the aircraft and traffic flow characteristic parameters, establishes an aircraft dynamics model; analyzes the structural characteristics of the PBN airway, extracts the structure and flight parameters, establishes the flight environment.

- A position detection and processing module: According to the integration data in real time, the dynamic ADS-B, the native aircraft status database, the ground air traffic control radar data and other relevant aircraft data, the aircraft position must be located. It can provide operational data to support the conflict detection module and the inference decision-making module.

- A conflict detection module: Displays traffic information by monitoring the situation around the aircraft and records operating data about the relevant aircraft for analytical reasoning. Updates the native and adjacent aircraft location based on the conducted data transmitted from the position detection and processing module, in order to predict possible conflicts and determine the degree of risk.

- An inference decision making module: It is the core part of the aircraft agent, which provides safe flight control for the aircraft on a PBN airway. The aircraft en route spacing is automatically adjusted, based on the interactive information obtained dynamically from the native and adjacent aircraft flight status database. The aircraft speed and state is adjusted in real time on the PBN airway. The inference decision making module analyzes the data transfer from the flightplanning, position-detection and processing, conflict-detection, flight-status module, according to the predetermined decision inference rules to determine the 
aircraft action to be performed. It specifies the state adjustment method to ensure absolute aircraft safety of the PBN airway.

- A flight status management module: includes a flight converter, regulator and monitor. The converter transforms the aircraft flying state based on the position-detection and processing module and inference decision making rules. A regulator executes the adjustment of the flight state issued by reasonable decision making. The monitor is responsible for monitoring the effect of the status adjustment. The adjustment information of the flight status is issued by the effector to other aircrafts.

- An information exchange module: using broadcast networks and satellite communications, through sensors and effectors, it exchanges and shares dynamic information with other aircrafts on a PBN airway in real-time. Exchanging information includes this aircraft and other aircraft flight data. The sensor collects information of other relevant aircrafts in real-time and transmits it to the position detection and processing module database. The effector releases this aircraft operational information, including the speed, location, operating status and other real time information. The module issues data, collecting dynamic information through a broadcast network, providing it to the position detection and processing, and inference decision making module. Position detection and processing module dynamically integrate, updating the PBN airway trend and provides a reference for the inference decision making module. The inference decision making module determines the aircraft state according to the relevant information and after the effector releases the latest information to the broadcast networks.

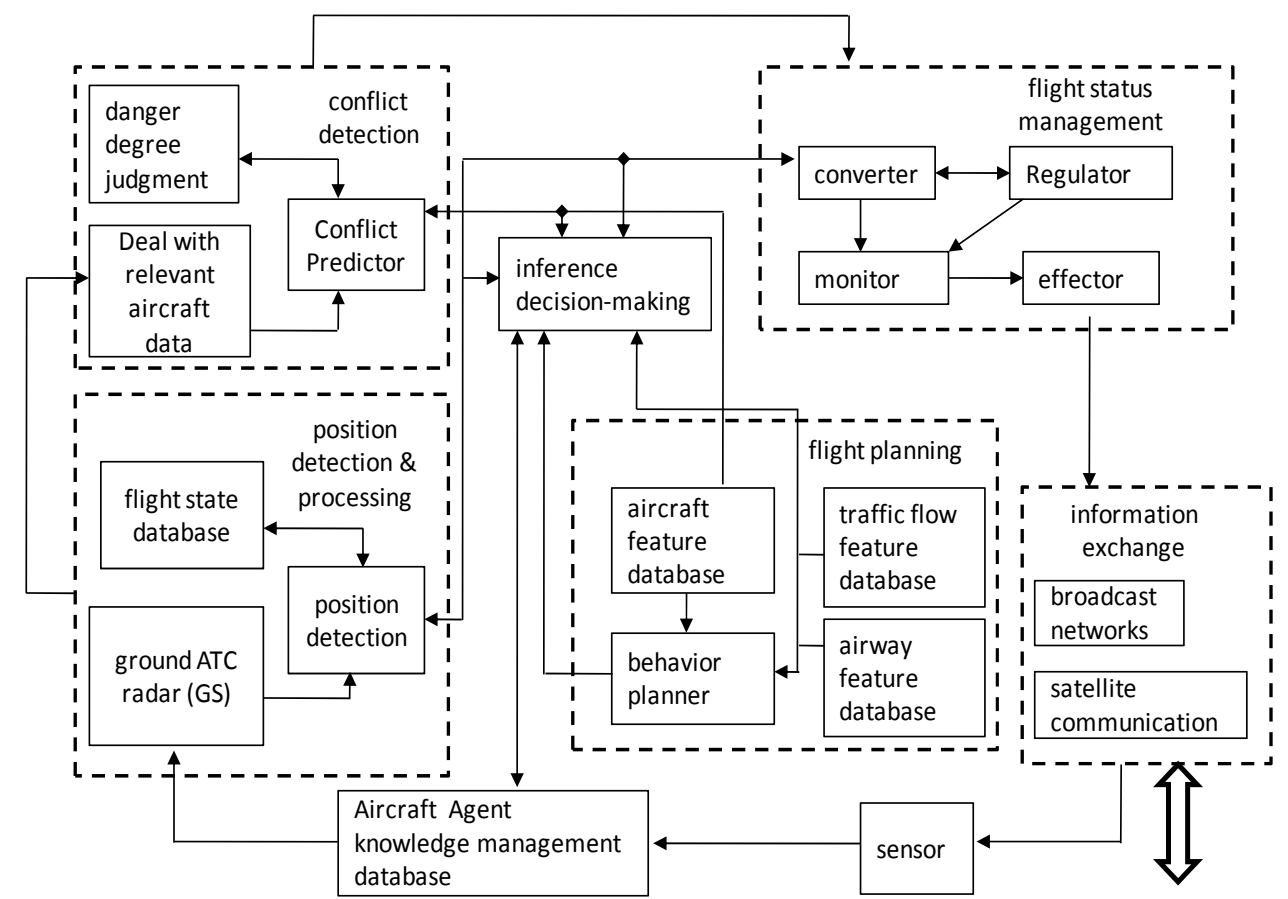

Fig. 1. Interior structure of the aircraft agent 


\subsection{The basic inference decision algorithm flow}

The aircraft agent begins to work at the starting point of the PBN airway and demises at the end of the airway. In the whole operation process, the information of the other aircraft and airway status are constantly acquired, and the flight state is dynamically adjusted. The aircraft flies along a preset airway by an autonomous flight system.

The flight states of the aircraft agent are called a target velocity state, velocity adjustment state, airway change state and airway out of state on a PBN airway. The target velocity state without considering the impact of the front aircraft is only according to its target velocity on the airway. The state of velocity adjustment means that the aircraft adjusts its acceleration and velocity according to the position and velocity of the front aircraft, controls the spacing of the front aircraft, achieves automatic interval management. If the adjustment parameters, based on the interval and velocity data have already exceeded the performance data of the aircraft, insufficient to achieve the safety interval, an airway change command will be issued and the aircraft taken into the airway change state. The execution needs certain time, meeting with an airway interval greater than a fixed value, or an aircraft into an airway out of state.

\section{Deceleration control based on safe separation and speed constraint}

Consider one front aircraft and one following aircraft on the same flight segment of the control area. The front aircraft is decelerated at time $t$, and the target speed is $v_{m}$; for the following aircraft, the speed has to be reduced to $v_{n+1}^{\text {last }}$. After deceleration of the front aircraft, suppose that the time of the air traffic controller reacting and making command is $t_{1}$, the time of the pilot execution of the command is $t_{2}$, the time of establishing the flying posture is $t_{3}$, and the total time $T=t_{1}+t_{2}+t_{3}$. Therefore, the following aircraft will not start deceleration until a delay of $T$. That is, the following aircraft is decelerated at $t+T$. The time $T$ is related to the work load of the air traffic controller and to the reaction of the pilot.

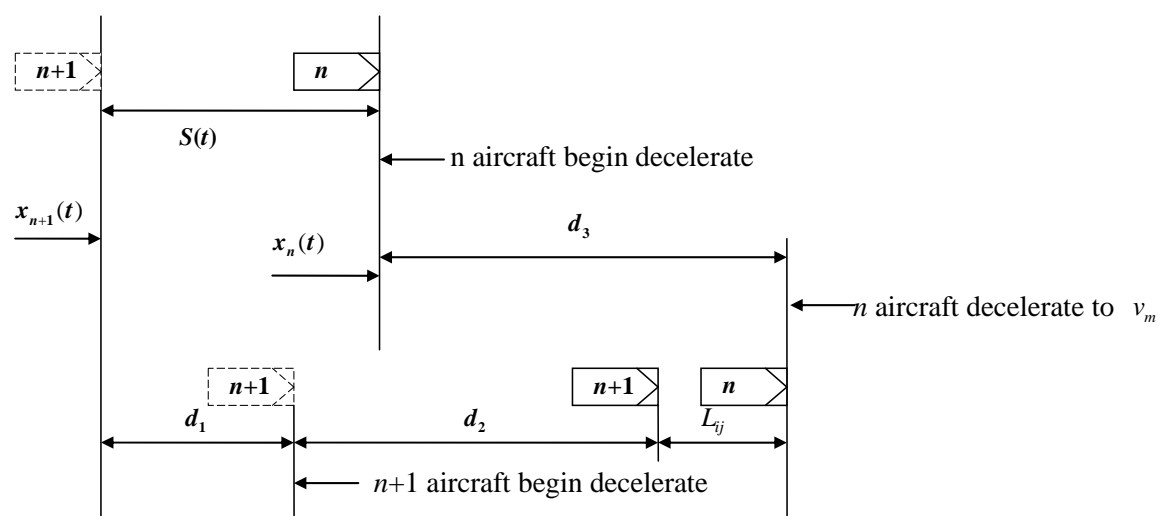

Fig. 2. Following model of an aircraft 
Fig. 2 presents the scheme of a flight following model. In the figure, $n$ denotes the front aircraft, and $n+1$ the following aircraft; $s(t)$ is the distance between the heads of the two aircrafts at $t ; v_{n}(t)$ and $v_{n+1}(t)$ are the flying speed of the front aircraft and the following aircraft at $t$, respectively; $d_{1}$ is the distance fly to the following aircraft before $T$, and $v_{n+1}(t)$ of the following aircraft remains constant within $T ; d_{2}$ is the distance fly to the following aircraft from the start of deceleration to the speed $v_{n+1}^{\text {last }} ; d_{3}$ is the distance fly by the front aircraft from the start of deceleration to the speed $v_{m} ; L_{i j}$ is the minimal tail vortex separation between the aircraft of type $i$ and the aircraft of type $j ; i$ and $j$ are the types of the front aircraft $n$ and the following aircraft $n+1$, respectively, $i, j \in\{H, M, L\}$. There are nine combinations of $i$ and $j$ (see Table 1). $a_{n}^{i}$ and $a_{n+1}^{j}$ are the deceleration of the front aircraft $n$ (type $i$ ) and the following aircraft $n+1$ (type $j$ ), respectively. Their values are negative in deceleration.

\subsection{The safe separation constraint}

From Fig. 2 the safe separation constraints are obtained as follows:

$$
\begin{gathered}
s(t)+d_{3} \geq d_{1}+d_{2}+L_{i j}, \\
d_{1}=v_{n+1}(t) T=v_{n+1}(t+T) T,
\end{gathered}
$$

$$
d_{2}=\frac{\left[\left(v_{n+1}^{\mathrm{last}}\right)^{2}-v_{n+1}^{2}(t)\right]}{2 a_{n+1}^{j 1}}
$$

$$
d_{3}=\frac{\left[v_{m}^{2}-v_{n}^{2}(t)\right]}{2 a_{n}^{i}},
$$

$$
s(t)+\frac{\left[v_{m}^{2}-v_{n}^{2}(t)\right]}{2 a_{n}^{i}} \geq v_{n+1}(t) T+\frac{\left[\left(v_{n+1}^{\text {last }}\right)^{2}-v_{n+1}^{2}(t)\right]}{2 a_{n+1}^{j 1}}+L_{i j} ;
$$

because $a_{n+1}^{j 1}<0$,

$$
\begin{gathered}
\frac{\left[\left(v_{n+1}^{\text {last }}\right)^{2}-v_{n+1}^{2}(t)\right]}{2 a_{n+1}^{j 1}}-\frac{\left[v_{m}^{2}-v_{n}^{2}(t)\right]}{2 a_{n}^{i}} \leq s(t)-v_{n+1}(t) T-L_{i j}, \\
a_{n+1}^{j 1} \leq \frac{\left[\left(v_{n+1}^{\text {last }}\right)^{2}-v_{n+1}^{2}(t)\right]}{2\left\{s(t)-v_{n+1}(t) T-L_{i j}+\frac{\left[v_{m}^{2}-v_{n}^{2}(t)\right]}{2 a_{n}^{i}}\right\}} .
\end{gathered}
$$

4.2. The speed constraint of the follow aircraft

To avoid a dangerous approach, the final speed of the following aircraft must be less or equal to the final speed of the front aircraft after deceleration, i.e., $v_{n+1}^{\text {last }} \leq v_{m}$. The aircrafts have to fly above the stalling speed. Therefore, $v_{m}>v_{\mathrm{s}}^{i}(n)$, 
$v_{n+1}^{\text {last }}>v_{s}^{j}(n+1)$ for the two aircrafts, where $v_{s}^{i}(n)$ and $v_{s}^{j}(n+1)$ are the stalling speed of the front aircraft $n$ (type $i$ ) and the following aircraft $n+1$ (type $j$ ), respectively. The speed constraints are expressed as follows:

$$
v_{n+1}^{\text {last }}=v_{n+1}(t)+a_{n+1}^{j 2}\left\{\frac{\left[v_{m}-v_{n}(t)\right]}{a_{n}^{i}}-T\right\} \leq v_{m},
$$

$T$ is subtracted from $\left[\left(v_{m}-v_{n}(t)\right] / a_{n}^{i}\right.$, which is the time taken for the front aircraft to decelerate to $v_{m} ;\left\{\frac{\left[v_{m}-v_{n}(t)\right]}{a_{n}^{i}}-T\right\}$ is the flight time of the follow aircraft. After sorting, is obtained

$$
a_{n+1}^{j 2} \leq \frac{v_{m}-v_{n+1}(t)}{\left[v_{m}-v_{n}(t)\right] / a_{n}^{i}-T}
$$

and

$$
\left\{\begin{aligned}
& a_{n+1}^{j 1} \leq \frac{\left[\left(v_{n+1}^{\text {last }}\right)^{2}-v_{n+1}^{2}(t)\right]}{2\left\{s(t)-v_{n+1}(t) T-L_{i j}+\left[v_{m}^{2}-v_{n}^{2}(t)\right] / 2 a_{n}^{i}\right\}}, \\
& a_{n+1}^{j 2} \leq \frac{v_{m}-v_{n+1}(t)}{\left[v_{m}-v_{n}(t)\right] / a_{n}^{i}-T}, \\
& v_{n+1}^{\text {last }}>v_{\mathrm{s}}^{j}(n+1), \\
& a_{n+1}^{j^{*}}=\min \left(a_{n+1}^{j 1}, a_{n+1}^{j 2}\right)>\left(a_{n+1}^{j}\right)_{\min } .
\end{aligned}\right.
$$

It can be seen from (10), that the reaction is influenced by the initial separation, reaction time, cruising speed of the aircrafts, speed after deceleration and deceleration (or duration of deceleration) of the front aircraft during deceleration. Obviously, $a_{n+1}^{j^{*}}=\min \left(a_{n+1}^{j 1}, a_{n+1}^{j 2}\right)$ of the following aircraft varies more intensively with the decrease of the initial separation. The longer the reaction time, the higher the deceleration of the following aircraft will be.

\section{Simulation}

The wake strength of the aircraft will be improved with the increase of its weight. The aircrafts are divided into three types - heavy, medium and light, according to the Maximum Take-Off Weight (MTOW). Their average approach speed and average length are shown in Table 1 . The wake vortex separation standard in American FAR and the wake separation standard in Chinese CAAR are shown in Table 2.

Table 1. Aircraft type and parameters

\begin{tabular}{|c|c|c|c|}
\hline Aircraft classification & Heavy $(H)$ & Medium $(M)$ & Light $(L)$ \\
\hline MTOW $(\mathrm{kg})$ & $\geq 136000$ & $7000-136000$ & $\leq 7000$ \\
\hline Average length of aircraft $L_{i}(\mathrm{~m})$ & 65 & 40 & 20 \\
\hline Average approach speed $(\mathrm{km} / \mathrm{h})$ & 270 & 237 & 177 \\
\hline
\end{tabular}


Table 2. FAR \& CCAR Minimal wake vortex separation standard

\begin{tabular}{|c|c|c|c|c|}
\hline \multirow{2}{*}{$\begin{array}{c}\text { Wake vortex separation } d_{i j} \\
\text { (FAR:nm/CCAR:km) }\end{array}$} & \multicolumn{3}{|c|}{ Follow aircraft } \\
\cline { 2 - 5 } & $H$ & Heavy & Medium & Light \\
\hline \multirow{3}{*}{ Front aircraft } & $M$ & $3 / 8$ & $5 / 10$ & $6 / 12$ \\
\cline { 2 - 5 } & $L$ & $3 / 6$ & $3 / 6$ & $4 / 10$ \\
\cline { 2 - 5 } & & $3 / 6$ & $3 / 6$ \\
\hline
\end{tabular}

As the aircrafts approach the runway, the speed range is similar for the heavy and the medium aircraft. The maximum deceleration for the two aircraft types is both $0.608 \mathrm{~m} . \mathrm{s}^{-2}$, and the minimum is $-0.780 \mathrm{~m} . \mathrm{s}^{-2}$.

One front aircraft and one following aircraft approaching the runway in the terminal area are considered. As to the initial speeds, the value of $v_{n+1}^{\text {last }}$ is equal to $v_{m}$ during calculation. Deceleration constraints under various conditions are derived from (7) and (9) (see Table 3). It is noted that the speed adjustment of the aircrafts needs to conform to the air traffic control regulations. The indicator air speed must be multiple to $20 \mathrm{~km} / \mathrm{h}$ (approximately $5.56 \mathrm{~m} / \mathrm{s}$ ); the Mach number is usually the multiple of 0.01 Mach number.

Simulation is carried out under different conditions. For example, condition 1: The initial separation $s(t)$ is $20 \mathrm{~km}$; the front aircraft is a heavy-duty aircraft, with speed $v_{n}(t)$ of $324 \mathrm{~km} / \mathrm{h}(90 \mathrm{~m} / \mathrm{s})$; the following aircraft is a medium-duty aircraft, with speed $v_{n+1}(t)$ of $360 \mathrm{~km} / \mathrm{h}(100 \mathrm{~m} / \mathrm{s})$. The front aircraft starts deceleration at $t$, and the deceleration $a_{n}^{i}$ is $-0.1 \mathrm{~m} . \mathrm{s}^{-2}$. After the deceleration is over, the speed of the front aircraft $v_{m}$ is $304 \mathrm{~km} / \mathrm{h}(84.4 \mathrm{~m} / \mathrm{s})$ and the reaction time $T$ is $10 \mathrm{~s}$. According to Table 1, the final separation distance after deceleration is $10 \mathrm{~km}$, and the deceleration of the follow aircraft is $-0.3391 \mathrm{~m} . \mathrm{s}^{-2}$.

Table 3. Following aircraft deceleration under different conditions

Table 3. Following aircraft deceleration under different conditions
\begin{tabular}{|c|c|c|c|c|c|c|c|c|c|c|}
\hline Condition & $n$ & $n+1$ & $\begin{array}{c}L_{i j}, \\
\mathrm{~km}\end{array}$ & $\begin{array}{c}s(t), \\
\mathrm{km}\end{array}$ & $\begin{array}{c}T, \\
\mathrm{~s}\end{array}$ & $\begin{array}{c}v_{n}(t), \\
\mathrm{m} / \mathrm{s}\end{array}$ & $\begin{array}{c}v_{m}, \\
\mathrm{~m} / \mathrm{s}\end{array}$ & $\begin{array}{c}v_{n+1}(t), \\
\mathrm{m} / \mathrm{s}\end{array}$ & $\begin{array}{c}a_{n}^{i}, \\
\mathrm{~m} . \mathrm{s}^{-2}\end{array}$ & $\begin{array}{c}a_{n+1}^{j *} \\
\mathrm{~m}_{\mathrm{s}} \mathrm{s}^{-2}\end{array}$ \\
\hline 1 & $H$ & $M$ & 10 & 20 & 10 & 90 & 84.4 & 100 & -0.1 & -0.3391 \\
\hline 2 & $H$ & $H$ & 8 & 20 & 8 & 120 & 108.9 & 130 & -0.1 & -0.2049 \\
\hline 3 & $M$ & $M$ & 6 & 20 & 10 & 100 & 88.9 & 110 & -0.15 & -0.3297 \\
\hline
\end{tabular}

\section{Summary}

The speed control of aircrafts on a PBN airway is an important function of the automatic flight management system. Drawing on the car-following theory, we build a speed control model for the following aircraft under simplified conditions.

Simulation analysis is carried out to investigate the microscopic and macroscopic influence of various parameters on the aircrafts flying on the path. To ensure non-congestion in the terminal area and to improve the safety, the aircrafts approaching the runway must react quickly and maintain appropriate safe separation and similar speed. The simulation shows that the model proposed is fit 
for safe separation estimation, air traffic management and air traffic capacity evaluation.

Acknowledgments: This work is funded by the National Natural Science Foundation of China (Grant Nos 71271113, U1233101) and Jiangsu Second Normal University Scientific Research Project (JSNU-Y-4606).

\section{References}

1. ICAO. Performance-Based Navigation (PBN) Manual (Doc9613), ICAO. 999 University Street, Montréal, Quebec, Canada H3C 5H7, 2008.

2. CAAC. CAAC 2009 Implementation Roadmap of Performance-Based Navigation. Beijing, China Civil Aviation Press, 2009. 10 p.

3. Tandale, M. D., P. Sengupta, P. K. Menon et al. Queuing Network Models of the National Airspace System. - In: Proc. of 26th Congress of International Council of the Aeronautical Sciences. Reston: AIAA, 2008, pp. 1-14.

4. H a n s e n, M. Micro-Level Analysis of Airport Delay Externalities Using Deterministic Queuing Models: A Case Study. - Journal of Air Transport Management, Vol. 8, 2002, No 2, pp. 73-87.

5. H a n s e n, M., A. O d o n i, D. L o vell et al. Advanced Stochastic Network Queuing Models of the Impact of 4d Trajectory Precision. - NEXTOR Research Symposium. Washington, DC, FAA Headquarters, 2008, pp. 1-25.

6. M e n o n, P. K., M. D. T a nd a le, J. K i m et al. A Framework for Stochastic Air Traffic Flow Modeling and Analysis. - In: Proc. of AIAA Guidance, Navigation, and Control Conference. Reston, AIAA, 2010, pp. 1-28.

7. B a y e n, A. M., R. L. R a f f a rd, C. J. T o m lin. Adjoint-Based Control of a New Eulerian Network Model of Air Traffic Flow. - IEEE Transactions on Control Systems Technology, Vol. 14, 2006, No 5, pp. 804-818.

8. R o b e li n, C. A., D. S u n, D. W u et al. MILP Control of Aggregate Eulerian Network Airspace Models. - In: Proc. of 2006 American Control Conference. Piscataway, NJ, IEEE, 2006, pp. 5257-5262.

9. Li g h thill, M. J., G. B. Whith a m. On Kinematic Waves. II. A Theory of Traffic Flow on Long Crowded Roads. - In: Proceedings of the Royal Society of London. Mathematical and Physical Sciences Series A, Vol. 229, 1955, No 1178, pp. 317-345.

10. D a g a $\mathrm{n}$ z o, C. F. The Cell Transmission Model, Part II: Network Traffic. - Transportation Research, Part B, Vol. 29, 1995, No 2, pp. 79-93.

11. Men on, P. K., G. D. S weriduk, K. B il i mori a. New Approach for Modeling, Analysis and Control of Air Traffic Flow. - AIAA Journal of Guidance, Control and Dynamics, Vol. 27, 2004, No 5, pp. 737-744.

12. Z h a n g, Z. N., M. C a i, L. L. W a ng et al. Research of Longitudinal Safety Separation Based on Nagel-Schreckenberg Traffic Flow Model. - Physics Procedia, Vol. 33, 2012, No 1, pp. 573-579.

13. Z h a n g, H. The Key Technologies of Collaborative Flow Management in Airport Terminal Area. Nanjing, Nanjing University of Aeronautics and Astronautics, 2009.

14. L i, X. H., Z. N. Z hang. Research of the Structure of Air Traffic Flow Management System Based on the Agent. - Journal of Transportation Engineering and Information, Vol. 5, 2007, No 1, pp. 56-61.

15. W a n g, W. L. Application of Multi-Agent System in Flight Conflict Detection and Resolution.Journal of Transport Information and Safety, Vol. 27, 2009, No 3, pp. 9-15.

16. D a i, L., X. Z. X i a. Application of Multi-Agent in Flight Conflict Resolution. - Ship Electronic Engineering, Vol. 28, 2009, No 3, pp. 62-64, 89.

17. W a n g, C., X. H. X u. Researching on Air Traffic System Using Agent-Based Modeling and Simulation. - Computer Engineering and Applications, Vol. 44, 2008, No 31, pp. 12-14. 
18. W a n g, F., X. H. X u, J. Z h a n g. Air Traffic Flow Collaborate Management Based on MultiAgent. - Journal of Guangxi Normal University: Natural Science Edition, Vol. 26, 2008, No 1, pp. 125-128.

19. Z h a n g, J. X., M. H. H u. Design of the Air Traffic Intelligent Simulation System for the Airport with Multi-Terminal Areas Based on Multi-Agents. - Journal of Transportation Engineering and Information, Vol. 7, 2009, No 2, pp. 90-98.

20. Hex m o or, H., T. Heng. Air Traffic Control Agents: Landing and Collision Avoidance. International Conference in Artificial Intelligence. Las Vegas, AIAA, 2000, pp. 21-35.

21. Nitschke, G. Cooperating Air Traffic Control Agents. - Applied Artificial Intelligence, Vol. 15, 2001, No 2, pp. 209-235.

22. C a llant ine, T. J. CATS-Based Air Traffic Controller Agents. Sacramento: NASA Ames Research Center, 2002.

23. Nguye n, M., J. Briot, A. Drogoul. An Application of Multi-Agent Coordination Techniques in Air Traffic Management. - In: Proc. of 2003 IEEE/WIC International Conference in Intelligent Agent Technology. Halifax, IEEE, 2003, pp. 622-625.

24. Hill, J., J. Archibald, W. Stirling et al. A Multi-Agent System Architecture for Distributed Air Traffic Control. - In: Proc. of 2005 AIAA Guidance, Navigation and Control Conference, San Francisco, AIAA, 2005, pp. 1005-1049.

25. A gogino, A., K. Tumer. Learning Indirect Actions in Complex Domains: Action Suggestions for Air Traffic Control. - Advances in Complex Systems, Vol. 12, 2009, No 4, pp. 493-512. 